\title{
Die Masseneinwanderungsinitiative gefährdet Ihre Gesundheit
}

\author{
Jürg Schlup \\ Dr. med., Präsident der FMH
}

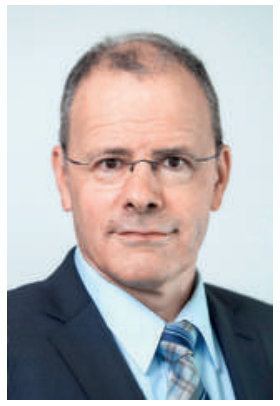

In fünf Monaten läuft die Frist zur Umsetzung der Masseneinwanderungsinitiative (MEI) ab, bislang ist dem Bundesrat aber - kaum überraschend - die Quadratur des Kreises, die ihm am 9. Februar 2014 aufgetragen wurde, noch nicht gelungen. Und als sei es nicht anspruchsvoll genug, die in der MEI geforderten Kontingente in Einklang mit der Personenfreizügigkeit zu bringen, erschwert nun auch noch der britische Austrittsentscheid die Verhandlungen. Sollte die vom Bundesrat angestrebte einvernehmliche Lösung mit der EU nicht gelingen und die Schweiz tatsächlich einseitige Massnahmen treffen, stünden die Bilateralen ernsthaft zur Disposition. Und so mutet es doch als Ironie der Geschichte an, dass der EU-Kommissions-Präsident Jean-Claude Juncker dieser Tage ausgerechnet anlässlich des 70. Jahrestags der Rede "Let Europe arise» von Winston Churchill an der Uni Zürich die Europa-ambivalente Schweiz besucht. Nach MEI und Brexit sowie inmitten der schweizerischen Diskussionen um Souveränität und EU-Rahmenabkommen dürfte ihm eine ähnlich visionäre Rede schwer fallen - so wichtig sie auch wäre.

\section{Von den jährlich neuen Fachärzten und -ärztinnen haben bereits seit 2006 über die Hälfte im Ausland studiert.}

Eine möglichst zukunftsfähige Umsetzung der MEI setzt aber nicht nur Visionen zur Europapolitik voraus, vor allem muss sie der Situation in der Schweiz gerecht werden. Nicht nur die Wirtschaft fürchtet den Kampf um Kontingente, besonders das Gesundheitswesen ist betroffen. In einem Land, in dem ein Drittel der berufstätigen Ärztinnen und Ärzte aus dem Ausland kommt, kann eine stärkere Beschränkung ihres Zuzuges die Versorgung limitieren. Von den jährlich neuen Fachärzten und -ärztinnen hat bereits seit 2006 die Mehrheit im Ausland studiert [1]. Im Jahr 2015 verfügten 70\% aller neuen Fachärzte und -ärztinnen über ein ausländisches Arztdiplom.
Wie unsere Gesundheitsversorgung aussieht, wenn zu wenige Ärzte und Ärztinnen aus dem Ausland kommen, sehen wir heute bereits am Hausärztemangel. Von unseren frischdiplomierten Ärztinnen und Ärzten wählen seit Jahrzehnten um die $40 \%$ Weiterbildungen in Grundversorgerdisziplinen [2], Tendenz eher steigend [1]. Dass uns dennoch Hausärzte und -ärztinnen fehlen, liegt daran, dass wir insgesamt viel zu wenige Nachwuchsärztinnen und -ärzte ausbilden. Sowohl bei Grundversorgern als auch bei Spezialisten sind wir auf Zuwanderung angewiesen, um unseren

\section{Sowohl bei Grundversorgern als auch} bei Spezialisten sind wir auf Zuwanderung angewiesen, um unseren Bedarf zu decken.

Bedarf zu decken. Weil aber die zuwandernden Kolleginnen und Kollegen häufiger Spezial- und seltener Grundversorgerdisziplinen ausüben, spüren wir bei den Spezialisten kaum Mangel - bei den Grundversorgern hingegen deutlich.

Da die seit 2009 stetig erfolgende Aufstockung der Medizinstudienplätze erst in einigen Jahren wirken kann, ist es für unsere Versorgungssicherheit unerlässlich, dass ausländische Ärztinnen und Ärzte weiterhin in die Schweiz kommen können. Einen bedarfsgerechten Fachärzte-Mix könnte eine Verschärfung der heutigen Qualitätskriterien für die Zulassung erreichen. Würde eine mindestens dreijährige Tätigkeit an einer schweizerischen Weiterbildungsstätte in der für die Zulassung beantragten Fachdisziplin zur Voraussetzung, entsprächen die neuen Zulassungen den verfügbaren Stellen mit fachspezifischer Tätigkeit - und damit etwa dem Bedarf

Das Gesundheitswesen zeigt - wie auch viele andere Branchen unseres Landes - dass die von der MEI angeblich geschützte Schweizer Unabhängigkeit eine Illusion ist. Inmitten Europas und in einer globalisierten Welt braucht die Schweiz Vernetzung und Austausch und die Patientenversorgung einen Bundesrat, dem die Quadratur des Kreises gelingt. 